\title{
Circuit
}

Musiques contemporaines

\section{Émergence de la forme dans la matière : de Varèse à Mozart}

\section{Gilles Tremblay}

Volume 6, numéro 1, 1995

Tremblay/Varèse/Messiaen : Gilles Tremblay analyste

URI : https://id.erudit.org/iderudit/902119ar

DOI : https://doi.org/10.7202/902119ar

Aller au sommaire du numéro

Éditeur(s)

Les Presses de l'Université de Montréal

ISSN

1183-1693 (imprimé)

1488-9692 (numérique)

Découvrir la revue

Citer cet article

Tremblay, G. (1995). Émergence de la forme dans la matière : de Varèse à

Mozart. Circuit, 6(1), 37-42. https://doi.org/10.7202/902119ar d'utilisation que vous pouvez consulter en ligne.

https://apropos.erudit.org/fr/usagers/politique-dutilisation/ 


\section{Émergence de la forme dans la matière : de Varèse à Mozart}

Conférence donnée au Conservatoire de Lyon dans le cadre des Rencontres du centre Jacques-Cartier, le 5 décembre 1989. Inédit.

$\|$ est téméraire pour un musicien d'aborder l'idée de matière et de forme devant des hommes de science et des philosophes. Toutefois, dans le cadre de notre échange, cette difficulté vaut largement d'être surmontée : le musicien (comme le danseur et le poète) n'a-t-il pas une connaissance toute particulière du temps, vivant et jouant intimement avec lui ? La matière et la forme peuventelles même se concevoir sans le temps, ce temps à la racine duquel jaillit le rythme? Cette notion même de rythme, source de la forme, sera point de jonction et connaissance partagée, chacun à sa manière, de pratique et de réflexion, par l'artiste et le savant. Rythme au sens le plus global, incluant l'idée de cycle, de l'infiniment petit (cycle des atomes) à l'infiniment grand (cycle des planètes, des galaxies, de la respiration de l'univers, d'explosions en implosions, d'élans en repos), ainsi que l'idée d'écoulement, donc de mouvement, que l'on trouve dans son étymologie même: de rheîn, couler.

Pourquoi ce rapprochement, qui peut paraître insolite, de Varèse et de Mozart ? Ce n'est ni par ce qu'ils ont en commun, ni par ce qui les opposent (du moins en apparence), mais plutôt par ce qu'ils apportent l'un et l'autre un éclairage révélateur à notre réflexion: Varèse aimait rappeler que, je cite, « notre maître c'est le son », et il a su tirer conséquence de la matière sonore dans la forme de ses œuvres; Mozart nous conduit plus globalement au plan du discours lui-même (d'un discours musical analogue au discours verbal, mais non sémantique), donc au plan du déroulement de la pensée et d'une faculté exceptionnelle de maîtriser le temps, comme en fait foi un texte étonnant que je citerai tout à l'heure, où il rapporte le processus de sa propre création.

Émergence de la forme dans la matière, on pourrait dire aussi émergence de la forme de la matière par la mise en vibration du son qui est rythme : matière ébranlée. L'émergence de la forme se trouve à la fois à l'intérieur de la matière par le mouvement des molécules qui sont vibrations et à l'extérieur par les creux et les pleins, les vides et les reliefs, pour utiliser un vocabulaire sculptural: « et il ne me reste qu'à couler la forme en va-et-vient, en creux en ronde-bosse », écrit Claude Ballif (1984, pp. 23-37) au sujet de son Coup de dés dans une lettre à Daniel Charles.

Je pense, bien avant Varèse, au début des Vêpres de 1610 de Monteverdi, où comme un fronton immense, un unique bloc sonore, l'accord de ré 
majeur est travaillé de l'intérieur et sur une longue durée; dans Beethoven, l'accord initial de la Pathétique, un bloc sonore, ébranle des faisceaux d'harmoniques non écrites mais audibles. En s'entrechoquant, elles déstabilisent l'accord générateur, le contredisent dramatiquement et affectent ainsi le caractère de l'œuvre entière; phénomène analogue dans son Appassionata aux interpolations hurlantes martelées gonflant la note d'appui du motif initial. La matière aura également une influence sur la forme lorsque la vitesse des vibrations, le registre, affectera les tempi, phénomène courant en électroacoustique, que Berlioz utilise prophétiquement dans la Symphonie fantastique en trois tempi différents sur le thème du Dies Iræ, large dans le grave, moyen au centre, rapide et grimaçant dans l'aigu. Stockhausen avait très justement observé dans ses cours de Darmstadt en 1957, que lorsque Mozart module à la dominante, cette modulation est très souvent associée à la présence de triolets : le rapport rythmique 3 contre 2 correspond exactement à l'intervalle de quinte, trois vibrations contre deux.

Debussy, de son côté, dissociera les différentes couches harmoniques, comme dans la Terrasse des audiences du clair de lune, où, à la fin de la section centrale, le registre moyen, délié des registres extrêmes, créera des vides ou des pleins dans la masse même du son.

Varèse, au début d'Intégrales, dissociera également en trois couches un bloc sonore extrêmement statique, mais renouvelé à chaque réitération par le mouvement rythmique, dynamique, et phonétique, toujours varié, informant chacune des strates sonores.

\section{Exemple 1}

Pte flute

Clarinette en si b

Pte flate

Pte clarinette si b

3 trombones

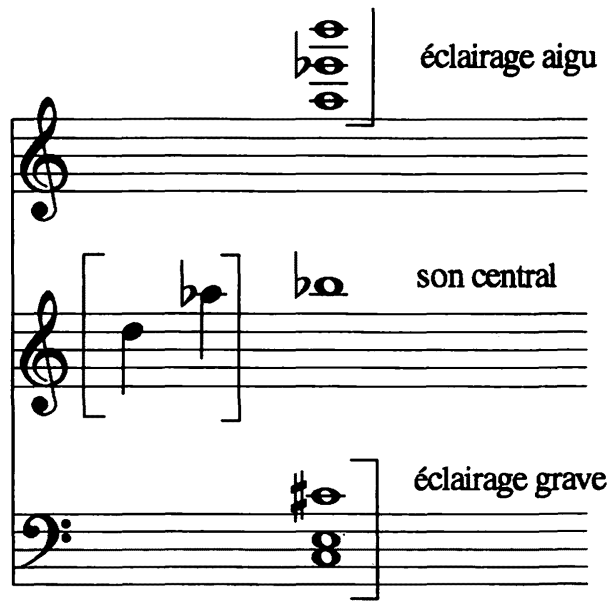

Varèse, Intégrales, bloc initial. 
Voici un autre exemple (minimal s'il en est) où l'on entend une forme mélodique s'extraire d'un son unique par les harmoniques naturelles qui en constituent l'échelle virtuelle. Ce son unique est ici chanté avec la technique dite de guimbarde, comme en témoigne ce chant de Mongolie.

\section{Exemple 2}

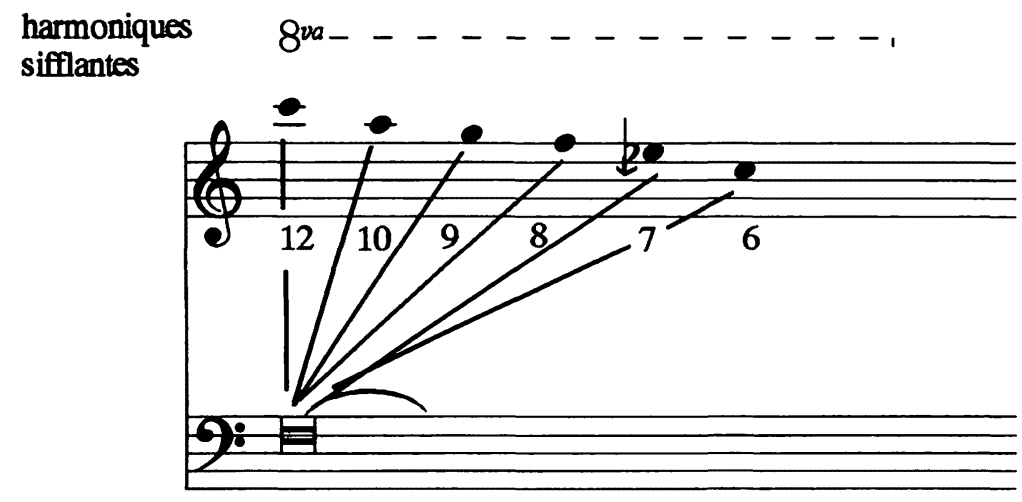

Mode pentaphone émergeant d'un « DRONE » unique (chant de Mongolie en guimbarde vocale).

Si l'on veut explorer un monde sonore encore plus microscopique, on n'aura qu'à augmenter démesurément des formes d'ondes complexes pour découvrir les abîmes et les crêtes vertigineuses qu'un appareil comme l'U.P.I.C., mis au point par Xenakis et son équipe, peut nous révéler. D'autre part, même à l'oreille nue, les harmoniques naturelles n'offrent-elles pas, comme les entendait Pythagore, la complexité croissante du nombre? Cette forme verticale à complexité croissante trouvera son équivalent horizontal (non systématique heureusement) dans la grande forme de cette même œuvre Intégrales, où les longues sections initiales se ramifient en une réexposition de plus en plus parcellaire à la fin du discours.

Voilà un mot-clé, discours, qui nous fait passer à un tout autre niveau. $\mathrm{Si}$ l'observation de la matière nous révèle des richesses insoupçonnées, cristaux, vagues ou nuages musicaux, la pensée discursive vole avec légèreté, met en relation, compare, organise, imagine, joue. Connaissant la matière, elle l'informe de façon conviviale et souveraine, enchante tout ce qu'elle touche. Elle ne s'enlise pas en elle, mais en fait émerger des formes qu'elle invente à l'infini, se nourrissant à toutes les terres et à toutes les lumières de la vie. Dans cette perspective, on aura compris qu'il ne s'agit point d'opposition entre une matière amorphe et une pensée formatrice pure et prométhéenne, mais d'une conjonction d'où naît l'œuvre, la forme n'étant pas l'enveloppe d'une matière violentée mais intimement liée à elle, à ses fibres mêmes. 
Trois composantes me semblent importantes dans l'élaboration de la forme musicale. Ce sont la mémoire, l'évolution et la constellation. La musique, rappelons-le, étant essentiellement un art du temps, c'est le fait que l'on puisse se souvenir, donc fixer des moments par la mémoire, qui a donné naissance à toutes les formes répétitives (litanies, musiques répétitives à cycles très courts chères aux écoles américaines récentes, ou aux cycles très longs et infiniment plus subtils des gamelans de Javal, ainsi qu'aux formes à refrain les plus diverses (depuis le simple A-B-A, jusqu'aux rondos les plus élaborés). Pratiquement toutes les formes classiques en sont issues, et on les trouve dans toutes les civilisations, ce qui en fait, à cause de leur universalité, de véritables archétypes formels. 1 On peut se demander si la notation qui permet de fixer la mémoire, de même que toutes les mémoires artificielles, n'ont pas paradoxalement un effet atrophiant sur cette capacité de notre cerveau - ce qui serait contraire au but recherché. C'est en tout cas une question, et une responsabilité, qui ne doit pas être éludée.)

Quant à l'évolution (seconde composante) elle permet de faire progresser l'œuvre à la manière d'un trait, sans aucun retour en arrière, en perpétuel renouvellement, variation pure, ouverte au méandre, au rêve, à ce qui advient; errance à l'écoute, prenant tous les risques de l'exploration. Énergie qui avance vers ce qui est autre, elle est sûrement une des plus fécondes en trouvailles de formes, et l'une des plus passionnantes de la démarche créatrice.

L'idée de constellation (troisième composante), correspond à un ensemble d'objets, sons, rythmes, timbres, événements pouvant être très éloignés les uns des autres (même hors du sonore : geste, couleur), mais unifiés par une harmonie (au sens large du mot) qui forme équilibre. Je pense à certaines séquences à éléments multiples dans Debussy, aux "Constellations-miroirs 》 de Boulez (Troisième sonate), aux mosaïques de tempi de Messiaen (notamment dans son Catalogue d'oiseaux: le "Traquet Stapazin», le * Merle de roche »), à plusieurs œuvres de Cage ainsi qu'aux ensembles statistiques de Xenakis.

Une des plus grandes difficultés du compositeur est que la musique étant un art du temps, les différents moments de l'œuvre n'existent pas simultanément, le moment présent reléguant, pendant l'élaboration, les moments passés et futurs dans l'ombre, sinon dans l'oubli, ce qui rend la conscience globale de l'œuvre extrêmement difficile. Or, voici ce que Mozart, qui, écrit Heidegger en guise de close à sa lettre (I), "fut l'un des plus oyant parmi ceux qui entendent " (fut, c'est-à-dire ne cesse d'être et toujours est), voici donc ce que Mozart nous dit aujourd'hui en ce 5 décembre, jour anniversaire de sa mort:

Parfois, au cours d'un voyage en voiture, ou bien après un bon dîner, en me promenant aussi dans la nuit quand je ne peux dormir, c'est alors que les pensées me viennent à flot et le mieux du monde. Celles qui à ce moment
(1) Extrait des lettres de Mozart, Das Musikleben, $j_{\text {ere }}$ année, $j^{\text {er }}$ cahier, Mayence 1948, cité in J. et B. Massin, Mozart, Paris, Fayard, 1970, vol. 1, p. 474.) 
m'agréent, je les retiens dans ma tête et me les fredonnent à moi-même, comme d'autres du moins m'ont dit de faire. Si je ne lâche pas prise, elles m'arrivent bientôt l'une après l'autre, et voilà un morceau à utiliser pour en faire un pâté suivant le contrepoint, suivant la sonorité des divers instruments, etc. Alors, cela me réchauffe l'âme si rien ne vient me déranger; cela ne cesse de grandir, je lui donne toujours plus d'ampleur, toujours plus de clarté, et la chose est en vérité presque faite dans ma tête, bien qu'il faille du temps pour qu'ensuite je l'embrasse d'une vue en esprit comme un beau tableau ou une charmante figure humaine et que j'entende en imagination, non plus ceci après cela, dans l'ordre où les choses ont bien pu venir, mais comme soudain tout à la fois. Cela est un régal! Tout, trouver et faire, advient maintenant en moi ni plus ni moins qu'en un beau rêve vigoureux. Mais embrasser de l'ouïe, ainsi tout à la fois, c'est bien ce qu'il y a de mieux.

« Embrasser de l'ouie ». Tout saisir, tout appréhender en une vision globale, avec tout ce que cela implique d'ouverture, de présence. À l'intérieur du présent, le pouvoir d'être accueillant, disponible à l'idée qui survient, imprévisible ${ }^{(2)}$, à l'émerveillement, à l'exclamation, au mouvementhors-de: émotion, émergence de la forme.

Ce que Mozart nous dit également, c'est ce don de rendre la durée simultanée dans l'instant, fulgurante... " comme soudain tout à la fois »; et son corollaire, car cette fulgurance n'est possible qu'associée d̀ la maturation, entourée par le long mûrissement de la gestation... « bien qu'il faille du temps »...

Patience dans l'azur ${ }^{(3)}$.
(2) * II'y a de création que dans l'imprévisible devenant nécessité • Pierre Boulez, son article «Éventuellement», la Revue musicale, $n^{\circ} 212$, Paris, avril 1952, p. 141.

(3) Titre d'un ouvrage d'Hubert Reeves, Patience dans l'azur : l'évolution cosmique.

BOULEZ, Pierre (1966), Relevés d'apprenti, Paris, Seuil.

CHARLES, Daniel (1984), « La poétique de Claude Ballif », Revue musicale, $n^{\circ}$ 370-371, pp. 23-37.

REEVES, Hubert (1981), Patience dans l'azur : l'évolution cosmique, Québec, $P V Q$. 


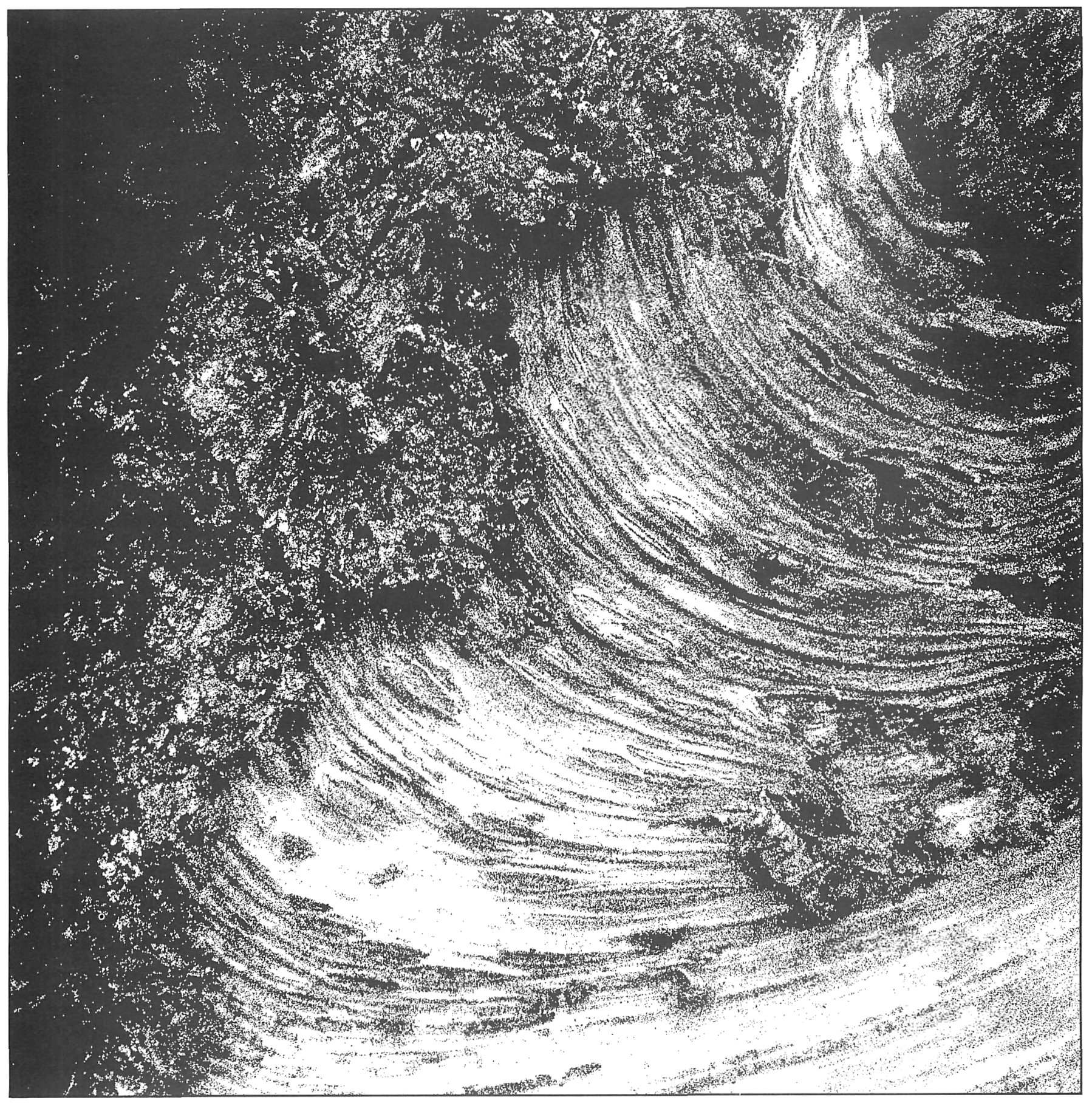

